\title{
A Parametrized Three-Dimensional Model for MEMS Thermal Shear-Stress Sensors
}

\author{
Qiao Lin, Yong Xu, Fukang Jiang, Yu-Chong Tai, Member, IEEE, and Chih-Ming Ho, Member, IEEE
}

\begin{abstract}
This paper presents an accurate and efficient model of MEMS thermal shear-stress sensors featuring a thin-film hotwire on a vacuum-isolated dielectric diaphragm. We consider three-dimensional (3-D) heat transfer in sensors operating in constant-temperature mode, and describe sensor response with a functional relationship between dimensionless forms of hotwire power and shear stress. This relationship is parametrized by the diaphragm aspect ratio and two additional dimensionless parameters that represent heat conduction in the hotwire and diaphragm. Closed-form correlations are obtained to represent this relationship, yielding a MEMS sensor model that is highly efficient while retaining the accuracy of three-dimensional heat transfer analysis. The model is compared with experimental data, and the agreement in the total and net hotwire power, the latter being a small second-order quantity induced by the applied shear stress, is respectively within $0.5 \%$ and $11 \%$ when uncertainties in sensor geometry and material properties are taken into account. The model is then used to elucidate thermal boundary layer characteristics for MEMS sensors, and in particular, quantitatively show that the relatively thick thermal boundary layer renders classical shear-stress sensor theory invalid for MEMS sensors operating in air. The model is also used to systematically study the effects of geometry and material properties on MEMS sensor behavior, yielding insights useful as practical design guidelines.

[1302]
\end{abstract}

\section{INTRODUCTION}

W HEN a fluid flows over a solid surface, viscous effects generate shear stress, or skin friction, on the surface. Knowledge of such wall shear stress is essential for understanding the dynamics of the fluid flow, and its measurement holds great importance for investigating and controlling wall-bound turbulence and flow separation [1]-[5]. Miniaturized shear-stress sensors fabricated using MEMS technology offer superior spatial resolution, minimized interference with fluid flow, and fast time response [6]. MEMS shear-stress sensors can be based on direct methods, typically using micromachined force-sensitive floating elements (e.g., [7], [8]) or indirect methods that primarily exploit thermal effects.

MEMS thermal shear-stress sensors are based on measurement of heat transfer from a heated thin-film element to the fluid flow. Since their initial demonstration [9], [10], much progress

Manuscript received March 15, 2004; revised June 24, 2004. Subject Editor S. M. Spearing.

Q. Lin is with the Department of Mechanical Engineering, Carnegie Mellon University, Pittsburgh, PA 15213 USA (e-mail: qlin@andrew.cmu.edu).

$\mathrm{Y}$. Xu is with the Department of Electrical and Computer Engineering, Wayne State University, Detroit, MI 48202 USA.

F. Jiang is with the Umachines, Altadena, CA 91001 USA.

Y.-C. Tai is with the Division of Engineering and Applied Science, California Institute of Technology, Pasadena, CA 91125 USA.

C.-M. Ho is with the Department of Mechanical and Aerospace Engineering, University of California, Los Angeles, CA 90024 USA.

Digital Object Identifier 10.1109/JMEMS.2005.844770 has been made in further development of MEMS thermal sensors for shear stress measurements. This includes sensors with alternative sensing element configurations and materials [11]-[15], and investigations of sensor characteristics such as thermal insulation, frequency response, pressure sensitivity and noise floor spectra [16]-[18]. In addition, MEMS shear-stress sensors have been fabricated in arrays on rigid [19] and flexible substrates [20], [21], integrated with signal conditioning circuitry [15], [20], [21], and applied to flow sensing and control in both air [22] and water [23]. Recently, there have also been numerical simulations of MEMS thermal shear-stress sensors to study effects of natural convection on fluid velocity profile [24] and effects of heat conduction in the sensor substrate on frequency response [25].

While these developments have been successful, a thorough understanding of the MEMS sensor operation has been lacking. In particular, it has been experimentally observed that the behavior of miniaturized shear-stress sensors often disagrees with classical shear-stress sensor theory, which states that the heat removed by the flow is proportional to the $1 / 3$-power of the shear stress [15]. We have performed systematic wind-tunnel testing of MEMS shear-stress sensors to study this issue, and interpreted the resulting experimental data qualitatively with a two-dimensional model [26]. While three-dimensional simulations were also performed, the numerical results were limited to the specific sensors tested and were not reusable if any material or geometric properties are varied from the values used in the simulations. To address the need for efficient and accurate evaluation of candidate sensor designs in iterative design processes, this paper presents a parametrized, three-dimensional (3-D) model, which is represented by closed-form expressions, for hotwire-based MEMS shear-stress sensors operating in constant-temperature mode. The model is validated with experimental data, and used to provide quantitative elucidation that the invalidity of classical shear-stress sensor theory for MEMS sensors is caused by the relatively large thickness of the thermal boundary layer on the sensor surface. Based on the model, the effects of sensor geometry and material properties on shear-stress sensing behavior are also systematically investigated.

The paper is organized as follows. The MEMS sensor configuration is described in Section II, and parametrized heat transfer equations are presented in Section III. The resulting model is experimentally validated in Section IV, and then used to elucidate thermal boundary layer characteristics and examine classical theory in Section V. Sections VI and VII, respectively, present a systematic study of the effects of sensor geometry and material properties on sensor behavior at zero and nonzero shear stress. 


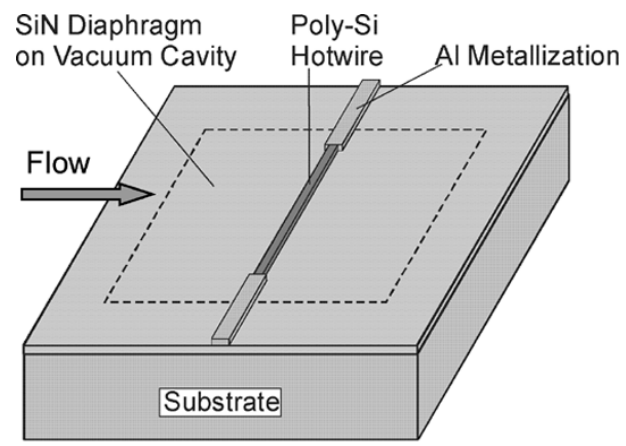

(a)

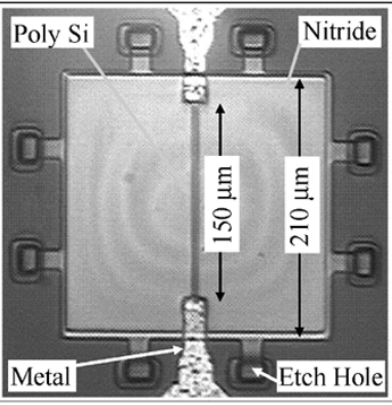

(b)

Fig. 1. (a) Schematic and (b) micrograph of a MEMS thermal shear-stress sensor.



Fig. 2. Illustration of the MEMS thermal shear-stress sensor model. Only one-half of the model is shown due to symmetry of the sensor about the $X Z$ plane.

A closed-form representation of the model is presented in Section VIII, and the conclusions from this work are finally given in Section IX.

\section{SENSOR CONFIGURATION}

We focus on a widely used class of MEMS shear-stress sensors that feature a resistive hotwire on a vacuum-isolated diaphragm [9], [10], [14], [19]-[21], [23]. A schematic and a micrograph of such sensors are shown in Fig. 1. The sensor consists of a resistive thin-film wire on a diaphragm, which in turn is suspended from the bulk of the substrate by a vacuum cavity. Aluminum metallization provides electrical connection to the resistor, which is electrically heated to function as a hotwire, with the vacuum providing enhanced thermal isolation. To measure wall shear stress, the sensor is mounted with the diaphragm surface flush with the wall and the hotwire perpendicular to the flow. In constant-temperature mode, the hotwire temperature is maintained constant, and the power consumed in the hotwire is measured to determine the shear stress.

\section{PARAMETRIZED MEMS SENSOR MODEL}

This section describes parametrized heat transfer equations for MEMS thermal shear-stress sensors. We focus on the sensor configuration described above; however, the modeling methodology is generally applicable to sensors with other configurations (e.g., [12], [15]). As illustrated in Fig. 2, the model considers a fluid flow over a vacuum-isolated thin diaphragm of length $2 L$, width $B$, and thickness $h$ (with $h \ll L$ and $h \ll B$ ).
A hotwire is embedded in the diaphragm along the diaphragm's plane of symmetry that is perpendicular to the flow, and spans the entire diaphragm width $B$. The hotwire has cross-sectional area $A_{w}$, with width $b_{w} \ll L$. As shown in the figure, we choose a coordinate frame with its origin at the center of the diaphragm surface, and its $\mathrm{X}$ and $\mathrm{Y}$ axes, respectively, aligned with the flow and hotwire.

A thermal boundary layer arises from hotwire heating on the sensor surface. This is a spatial region in which the fluid temperature rises above the ambient temperature (i.e., the temperature of the undisturbed bulk fluid). Because of the miniature MEMS sensor size, we adopt the assumption, which is generally made for conventional shear-stress sensors [1]-[3], that the entire thermal boundary layer is contained in the viscous sublayer of the velocity boundary layer. Therefore, the fluid velocity, $U$, and shear stress, $S$, which may vary with time (as in a turbulent flow), are related by a linear relationship in which $\mu_{f}$ is the fluid's dynamic viscosity [3]

$$
U=\frac{S Z}{\mu_{f}} .
$$

We consider heat transfer in the fluid, hotwire and diaphragm, whose temperatures are respectively $T_{f}, T_{w}$ and $T_{d}$. Define dimensionless temperatures by

$$
\theta_{f}=\frac{T_{f}-T_{\mathrm{amb}}}{\Theta_{\mathrm{w}}}, \quad \theta_{w}=\frac{T_{w}-T_{\mathrm{amb}}}{\Theta_{\mathrm{w}}}, \quad \theta_{d}=\frac{T_{d}-T_{\mathrm{amb}}}{\Theta_{\mathrm{w}}}
$$

and dimensionless shear stress $\tau$ and power $p$ by

$$
\tau=\frac{S L^{2}}{\mu_{f} \alpha_{f}}, \quad p=\frac{P}{k_{f} \Theta_{w} B}
$$

where $P$ is the power required to maintain the average hotwire temperature at a constant: $\Theta_{w}=1 / B \int_{-B / 2}^{B / 2}\left(T_{w}-T_{\mathrm{amb}}\right) d y=$ const. Also define the dimensionless thermal conductivities of the hotwire and diaphragm, $\sigma_{w}$ and $\sigma_{d}$, and the diaphragm aspect ratio (AR) by

$$
\sigma_{w}=\frac{k_{w} A_{w}}{k_{f} L^{2}}, \quad \sigma_{d}=\frac{k_{d} h}{k_{f} L}, \quad \mathrm{AR}=\frac{B}{2 L}
$$

where $k_{f}$ and $\alpha_{f}$ are the fluid's thermal conductivity and diffusivity, and $k_{w}$ and $k_{d}$ are the thermal conductivities of the hotwire and diaphragm. 
To obtain heat transfer governing equations, assume that spatial distributions of $T_{w}$ and $T_{d}$, are one- and two-dimensional, respectively. For simplicity, all thermal properties are assumed to be independent of temperature. Also, if the flow is timevarying, such variations are assumed to be sufficiently slow to allow use of steady-state heat transfer theory. Then, with dimensionless variables $x=X / L, y=Y / L$ and $z=Z / L$, heat conduction in the hotwire is governed by

$$
\begin{aligned}
& \sigma_{w} \frac{d^{2} \theta_{w}}{d y^{2}}+\sigma_{d}\left(\left.\frac{\partial \theta_{d}}{\partial x}\right|_{x=0+}-\left.\frac{\partial \theta_{d}}{\partial x}\right|_{x=0-}\right)+p=0 \\
& \left.\theta_{w}\right|_{y= \pm \mathrm{AR}}=0, \quad \frac{1}{2 \mathrm{AR}} \int_{-\mathrm{AR}}^{\mathrm{AR}} \theta_{w} d y=1
\end{aligned}
$$

where the heat loss directly from the hotwire to the fluid has been ignored using the approximation (further discussed in Section IV) of a vanishingly small hotwire width with the hotwire cross-sectional area kept constant. Heat conduction in the diaphragm is governed by

$$
\begin{aligned}
& \sigma_{d}\left(\frac{\partial^{2} \theta_{d}}{\partial x^{2}}+\frac{\partial^{2} \theta_{d}}{\partial y^{2}}\right)+\left.\frac{\partial \theta_{f}}{\partial z}\right|_{z=0}=0 \\
& \left.\theta_{d}\right|_{\Gamma_{d}}= \begin{cases}\theta_{w} & \text { if } x=0 \\
0 & \text { if otherwise }\end{cases}
\end{aligned}
$$

where $\Gamma_{d}$ denotes the diaphragm's edges. Finally, forced convection in the fluid is described by

$$
\begin{aligned}
& \tau z \frac{\partial \theta_{f}}{\partial x}=\frac{\partial^{2} \theta_{f}}{\partial x^{2}}+\frac{\partial^{2} \theta_{f}}{\partial y^{2}}+\frac{\partial^{2} \theta_{f}}{\partial z^{2}} \\
& \left.\theta_{f}\right|_{z=0}= \begin{cases}\theta_{d} & \text { if }-1 \leq x \leq 1, \quad-\mathrm{AR} \leq y \leq \mathrm{AR} \\
0 & \text { otherwise }\end{cases} \\
& \theta_{f} \rightarrow 0 \text { as } x \rightarrow \pm \infty, \quad y \rightarrow \pm \infty, \quad z \rightarrow \infty
\end{aligned}
$$

The boundary conditions in these equations assume that the substrate is an ideal heat sink at ambient temperature, as silicon is an excellent thermal conductor. This is supported by our measurements of substrate temperature immediately adjacent to the diaphragm and hotwire, which did not exceed $1^{\circ} \mathrm{C}$ above ambient. The effects of heat radiation and natural convection have also been neglected in these equations as they are considered insignificant [26].

A model for the MEMS thermal shear-stress sensors, given by the functional relationship $p=p(\tau)$, is thus obtained by numerically solving (5)-(7) using the ABAQUS finite element package. It will be convenient to further decompose $p$ in the form

$$
p=p_{0}+\Delta p
$$

where $p_{0}=p(0)$ is the dimensionless hotwire power in still fluid (i.e., $\tau=0$ ), and $\Delta_{p}=p-p_{0}$ is the dimensionless net hotwire power that is exclusively induced by nonzero shear stress.

\section{EXPERIMENTAL VALIDATION}

In this section we validate the model with wind-tunnel experimental data obtained from three MEMS shear-stress sensors [see Fig. 1(b)]. Details of the experiment are given elsewhere [26]. These sensors, referred to as Sensors 1, 2, and 3, each had a polysilicon hotwire $150-\mu \mathrm{m}$ long and $0.5-\mu \mathrm{m}$ thick, and a silicon-nitride diaphragm with dimensions $210 \times 210 \mu \mathrm{m}^{2}$ in the chip plane. The hotwire width and diaphragm thickness were 3 and $1.5 \mu \mathrm{m}$ (Sensor 1), 7 and $1.5 \mu \mathrm{m}$ (Sensor 2), and 7 and $3.0 \mu \mathrm{m}$ (Sensor 3), respectively. Operating in constant-temperature mode at $\Theta_{w}=118,93.4$, and $93.4^{\circ} \mathrm{C}$, the sensors respectively consumed $5.08,5.40$, and $11.25 \mathrm{~mW}$ of power in still air, corresponding to a dimensionless still-air hotwire power $\left(p_{0}\right)$ of $6.74,9.30$, and 19.4 (with thermal properties of air evaluated at $T_{\text {avg }}$ given below). The maximum shear stress measured in the experiment was $1.04 \mathrm{~Pa}$, corresponding to a dimensionless shear-stress $(\tau)$ of 17.4 (Sensor 1) and 19.1 (Sensors 2 and 3). With this correspondence between dimensional and dimensionless quantities for the sensors tested, we will exclusively use dimensionless shear stress and power in the remainder of this paper.

To evaluate the dimensionless parameters for the sensors tested, thermal properties of air are evaluated at the average temperature $T_{\mathrm{av}}=\left(T_{\mathrm{amb}}+T_{w}\right) / 2=T_{\mathrm{amb}}+\Theta_{w} / 2$ (with $T_{\mathrm{amb}}=25^{\circ} \mathrm{C}$ ) from published data [27], and the thermal conductivities of polysilicon and silicon nitride are given nominal values $k_{w}=34 \mathrm{~W} / \mathrm{m} \cdot \mathrm{K}$ and $k_{d}=3.2 \mathrm{~W} / \mathrm{m} \cdot \mathrm{K}$, respectively. The parameters $\left(\sigma_{w}, \sigma_{d}, \mathrm{AR}\right)$ are calculated to be $(0.194,1.50$, $1),(0.467,1.54,1)$, and $(0.570,3.09,1)$ for Sensors 1,2 , and 3 , respectively. Based on these nominal parameter values, the dimensionless hotwire power $p$ calculated from the model has an error of $16 \%, 4.4 \%$, and $20 \%$ for the three sensors when compared with experimental data, while the calculated dimensionless net hotwire power $\Delta p$ deviates from the experimental data by $28 \%, 37 \%$ and $35 \%$ for the three sensors.

These errors can be attributed to several nonidealities not considered in the model. The first factor is the uncertainty in material and geometric properties. In particular, the thermal conductivities of LPCVD polysilicon and silicon nitride are process-dependent, and their values reported in different sources differ by more than $100 \%$ [28]-[31]. Chip-to-chip variations in thicknesses of such thin films are on the order of $20 \%$. From (4), the uncertainty in the dimensionless conductivities $\sigma_{w}$ and $\sigma_{d}$ is of the same order. Additionally, as the hotwire does not span the entire diaphragm as assumed (see Fig. 1), the effective aspect ratio is expected to lie between 1 (the nominal value) and $150 / 210=0.71$ (the ratio of hotwire to diaphragm lengths). There are also other unmodeled effects such as temperature dependence of material properties, and natural convection and radiation effects [26].

Modeling error can also be caused by the neglect of heat transfer directly from hotwire to air. This is based on the assumption that when the total diaphragm length $(2 L)$, hotwire cross-sectional area $\left(A_{w}\right)$ and hotwire temperature $\left(\Theta_{w}\right)$ are held constant, the combined heat loss $\left(P_{d w}\right)$ directly from the diaphragm and hotwire surfaces to air is insensitive to hotwire width $b_{w} \ll L$. Thus, $b_{w}$ can be made vanishingly small and dropped from consideration, reducing by one the number of independent parameters in the model. We examine this assumption using Sensor 2 as an example. With $\tau$ ranging from 0 to 20 , $P_{d w}$ changes only by less than $3.4 \%$ as $b_{w}$ is decreased by two 


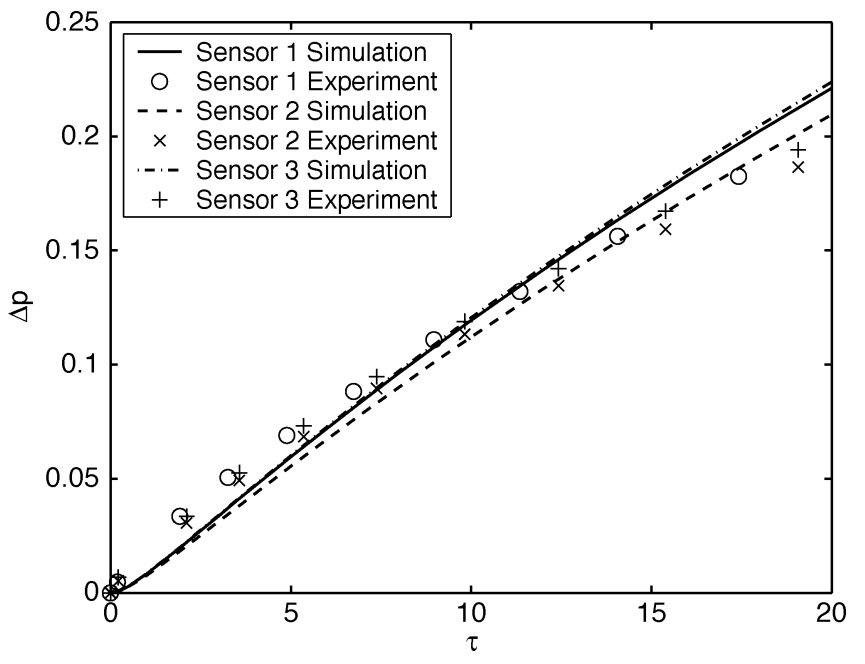

Fig. 3. Comparison of model-predicted and experimentally determined dimensionless net hotwire power $\Delta p=p-p_{0}$ as a function of dimensionless shear-stress $\tau$. For Sensors 1, 2, and 3, $p_{0}=6.74,9.30$ and 19.4 from the experiment, and $p_{0}=6.73,9.31$, and 19.4 from the model.

orders of magnitude from $7 \mu \mathrm{m}$ (the actual value) to a theoretical value of $0.07 \mu \mathrm{m}$ (or $b_{w} / L=1 / 1500$ ). The corresponding changes in $p$ and $\Delta p$ are respectively less than $1.2 \%$ and $6.2 \%$. That is, the effect of finite hotwire width is negligible on $p$, and small on $\Delta p$.

The uncertainty above can be approximately reflected in model predictions by adjusting the parameters $\sigma_{w}, \sigma_{d}$ and AR from their nominal values. To this end the sensitivity of sensor response to these parameters is first evaluated. Sensor 2 is used as an example, and the other sensors yield similar results. For $0<\tau<20$ and at $\left(\sigma_{w}, \sigma_{d}, \mathrm{AR}\right)=(0.467,1.54,1)$, the sensitivity ${ }^{1}$ of the total hotwire power $p$ is found to range from 0.16 to 0.17 with respect to $\sigma_{w}$, from 0.68 to 0.70 with respect to $\sigma_{d}$, and from -0.86 to -0.81 with respect to AR. That is, all three parameters significantly influence $p$. On the other hand, the corresponding sensitivity of the dimensionless net hotwire power $\Delta p$ ranges from 0.013 to 0.017 with respect to $\sigma_{w}$ and from 0.048 to 0.070 with respect to $\sigma_{d}$, but lies between 0.93 and 1.4 with respect to AR. Thus, for these sensors, $\Delta p$ is sensitive to changes in AR, but not to changes in the dimensionless conductivities.

Therefore, in parameter adjustment, we first adjust AR to reduce modeling error in $\Delta p$, and then $\sigma_{w}$ and $\sigma_{d}$ to reduce modeling error in $p$. The resulting adjusted values for $\left(\sigma_{w}, \sigma_{d}, \mathrm{AR}\right)$ are $(0.140,1.09,0.863),(0.400,1.28,0.804)$ and $(0.600,3.27$, 0.825 ) for Sensors 1,2 , and 3, respectively. These adjustments are consistent with the parameter uncertainty discussed above. The total hotwire power $p$ thus predicted agrees with the experimental data within $0.4 \%$ at all measured shear stresses. Discrepancies between the model-predicted net hotwire power $\Delta p$ and experimental data is also reduced, for all tested sensors, to $11 \%$ or less for $\tau>6$, when forced convection is sufficiently significant (see Fig. 3). This error, while larger than that for $p$, is considered acceptable as $\Delta p$ represents second-order heat

${ }^{1}$ The sensitivity of a quantity $q$ with respect to some parameter $s$ is defined as $(\partial q / \partial s) /(q / s)$, which gives the relative change of $q$ produced by a given relative change of $s$. transfer effects and accounts for only about $3 \%$ or less of the total hotwire power. More accurate modeling of the nonidealities above should further reduce the error.

Finally, Fig. 3 affords an interesting insight into the three sensors tested, which had different hotwire cross-sectional dimensions and diaphragm thicknesses, and operated at different temperatures. The relations between $\tau$ and $\Delta p$ for these sensors, both from the model and experiment, almost collapse to a single curve. Thus, the model indeed captures the trend in the experimental data. In particular, as the sensors had the same AR but different $\sigma_{w}$ and $\sigma_{d}$ values, we observe that for these sensors, AR plays a major role in determining $\Delta p$, while $\sigma_{w}$ and $\sigma_{d}$ do not. This observation is consistent with the sensitivity analysis above, and as will be shown in Section VII, reflects the general insensitivity of $\Delta p$ to $\sigma_{w}$ and the saturated dependence of $\Delta p$ on $\sigma_{d}$.

\section{THERMAL BOUNDARY LAYER CHARACTERISTICS AND INADEQUACY OF CLASSICAL THEORY}

In classical theory, the sensor is assumed to be a constant-temperature flat plate with an infinite spanwise dimension, and the thermal boundary layer is assumed to be thin compared with the plate length [3]. Heat transfer in the fluid is then dominated by forced convection and conduction across the boundary layer. Other heat conduction contributions are ignored. Applied to MEMS shear-stress sensors, the plate length is taken to be the diaphragm length $(2 L)$. In the dimensionless parameters defined in (3) and (8), this yields $\Delta p=1.28 \tau^{1 / 3}$, i.e., the net hotwire power is proportional to the $1 / 3$-power of the shear stress.

This classical relationship has been used for MEMS shearstress sensors due to a lack of a micro sensor model [13], [15], [18], [19]. It is important, however, to note that this law is valid only if the thermal boundary layer is thin; that is, $\delta \ll L$, where $\delta$ is the maximum thermal boundary layer thickness over the heated plate. As $\delta / L=3.68 \tau^{-1 / 3}$ [3], this requirement translates into $\tau \gg 50$. Applied to (MEMS or conventional) hot-film sensors that are used in air flow, the diaphragm half-length must satisfy the condition

$$
L \gg \frac{175}{\sqrt{S}} \mu \mathrm{m}
$$

where $S$ has the unit of $\mathrm{Pa}$, and thermal properties of air are representatively evaluated at $75^{\circ} \mathrm{C}$.

It can be seen that this condition is generally not satisfied by MEMS thermal shear-stress sensors, with $L$ on the order of hundreds of microns or less, for air flow measurements that typically involve shear stresses on the order of $1 \mathrm{~Pa}$. In particular, the sensors tested $(L=105 \mu \mathrm{m})$ in our experiment violate this requirement. The experimental data (Fig. 3) indeed deviates significantly from the classical power law, and instead is described approximately by $\Delta p \propto \tau^{0.77}$. This is correctly predicted by the three-dimensional model, which approximately gives $\Delta p \propto \tau^{0.79}$ for $1 \leq \tau \leq 24$ for all three sensors tested. The 0.79-power law agrees with the experimental data more closely than the 0.67 -power law as predicted by a more simplified, two-dimensional (2-D) analysis [26]. 


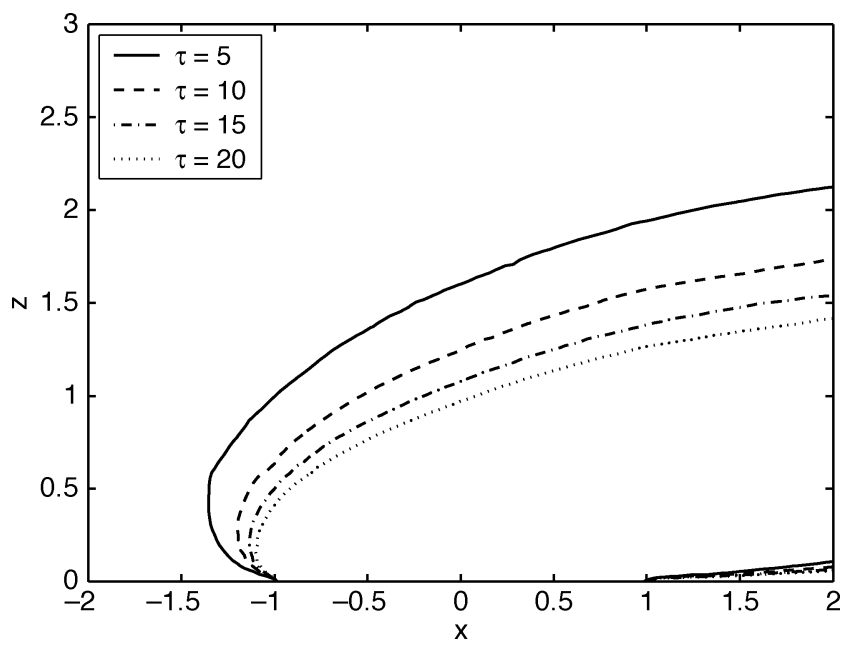

Fig. 4. Edge of the thermal boundary layer (on which $\theta_{f}=0.01$ ) for Sensor 2 at different shear-stress values plotted in dimensionless spatial coordinates. The diaphragm spans the interval $[-11]$ on the $\mathrm{x}$-axis.

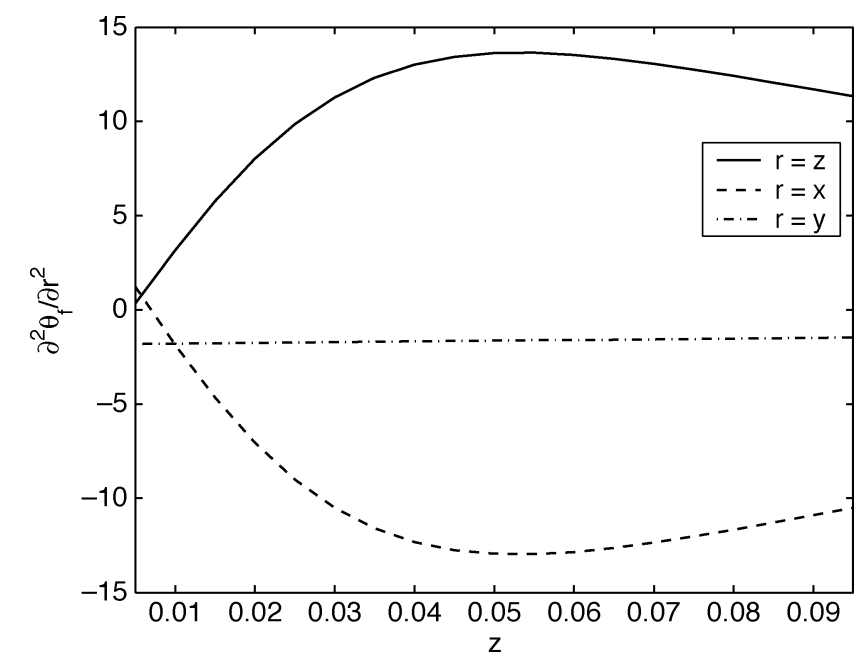

Fig. 5. Second-order partial derivatives of the fluid temperature along the $\mathrm{Z}$-axis $(\tau=20$ for Sensor 2).

The MEMS shear-stress sensor model allows quantitative insight into the characteristics of the thermal boundary layer. We consider Sensor 2 with the nominal parameter values $\left(\sigma_{w}, \sigma_{d}, \mathrm{AR}\right)=(0.467,1.54,1)$. The edge of the thermal boundary layer, at which the temperature has decreased to $1 \%$ of the average hotwire temperature, is computed from the model and shown in Fig. 4 for $\tau=5,10,15$, and 20. It can be seen that the thermal boundary layer is not thin, with a thickness comparable to the diaphragm length for all these shear-stress values.

Also, streamwise heat conduction in the fluid, which is ignored in classical theory, can be considered to gain insight into the boundary layer. Again using Sensor 2 as an example, Fig. 5 shows second-order partial derivatives of fluid temperature $\theta_{f}(\tau=20)$, which represent heat conduction in three dimensions, along the z-axis (Fig. 2). We can see that $\partial^{2} \theta_{f} / \partial x^{2}$ is comparable in magnitude with $\partial^{2} \theta_{f} / \partial z^{2}$, indicating that streamwise heat conduction is as significant as heat conduction across the thermal boundary layer, and hence cannot be ignored.

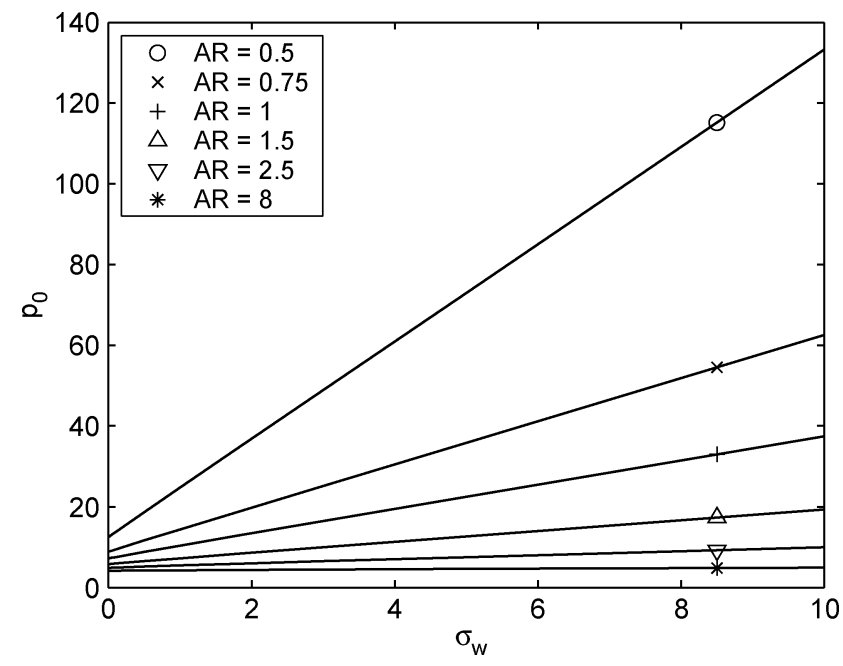

Fig. 6. Dependence of the dimensionless still-fluid hotwire power $p_{0}$ on the dimensionless hotwire conductivity $\sigma_{w}$ for different ARs $\left(\sigma_{d}=1.5\right)$.

\section{SENSOR RESPONSE IN STILl FLUID}

The parametrized model allows us to systematically study the effects of the parameters $\sigma_{w}, \sigma_{d}$ and AR on sensor behavior. This section considers such effects on the still-fluid hotwire power $\left(p_{0}\right)$, and the next section will consider the net hotwire power $(\Delta p)$. We compute $p_{0}$ by numerically solving $(5)-(7)$ with $\tau=0$, with the three parameters varied from the reference values $\sigma_{w}=0.5, \sigma_{d}=1.5$ and $\mathrm{AR}=1$, which approximately represents Sensor 2.

Fig. 6 depicts the dependence of $p_{0}$ on the dimensionless hotwire conductivity $\sigma_{w}$ as $\mathrm{AR}$ is varied. It can be seen that $p_{0}$ increases virtually linearly in the entire range of $\sigma_{w}$, and decreases with $\mathrm{AR}$ at fixed $\sigma_{w}$. In addition, the slope $\partial p_{0} / \partial \sigma_{w}$ decreases as AR increases, until $p_{0}$ becomes virtually independent of $\sigma_{w}$. This effect of AR agrees with the intuition that the conduction heat loss to the substrate at the hotwire ends and streamwise diaphragm edges decreases with AR and becomes diminished at sufficiently large AR, when the sensor behavior becomes 2-D. It is also intuitively clear that $p_{0}$ increases with $\sigma_{w}$, as higher hotwire conductivity leads to greater power consumption. To explain the nearly linear relationship between $p_{0}$ and $\sigma_{w}$, Fig. 7 shows the temperature distributions along the hotwire and the streamwise centerline of the diaphragm. As $\sigma_{w}$ varies by two orders of magnitude from 0.05 to $0.5, \theta_{w}$ and $\theta_{d}$ change only by less than $15 \%$ with respect to the average hotwire temperature. As the hotwire and diaphragm temperatures are insensitive to $\sigma_{w},(5)$ indicates that $p_{0}$ will be approximately linear in $\sigma_{w}$.

The effect of the dimensionless diaphragm conductivity $\sigma_{d}$ on the still-air power $p_{0}$ is shown in Fig. 8. First note that for fixed $\sigma_{d}, p_{0}$ again decreases with AR. For a given AR, $p_{0}$ increases with $\sigma_{d}$, as there is more heat loss to the substrate through a diaphragm of higher conductivity. It can also be seen in Fig. 8 that while $p_{0}$ increases nonlinearly with $\sigma_{d}$ when $\sigma_{d}$ is small, the dependence becomes almost linear when $\sigma_{d}$ is sufficiently large (approximately $\sigma_{d}>0.15$; also see Section VIII). This is because for sufficiently large $\sigma_{d}$, there is significant heat conduction in the diaphragm. The temperature distributions in the 




Fig. 7. Normalized temperature distributions along the hotwire and streamwise centerline of the diaphragm for different values of the dimensionless hotwire conductivity $\sigma_{w}\left(\sigma_{d}=1.5, \mathrm{AR}=1\right)$.



Fig. 8. Dependence of the dimensionless still-fluid hotwire power $p_{0}$ on the dimensionless diaphragm conductivity $\sigma_{d}$ for different ARs $\left(\sigma_{w}=0.5\right)$.

hotwire and diaphragm in this case become insensitive to the specific value of $\sigma_{d}$, in a manner similar to that illustrated in Fig. 7. Such insensitivity then implies, again according to (5), that $p_{0}$ is approximately linear in $\sigma_{d}$.

\section{SENSOR RESPONSE TO SHEAR STRESS}

We consider $\Delta p$, the dimensionless net hotwire power induced by applied shear-stress, as a function of the dimensionless shear stress $\tau$. This function in general depends on $\sigma_{w}, \sigma_{d}$, and $\mathrm{AR}$, which will now be varied one at a time from the reference values $\sigma_{w}=0.5, \sigma_{d}=1.5$ and $\mathrm{AR}=1$. Note that when these parameters are fixed, $\Delta p$ always increases with $\tau$ as a result of increased convection heat transfer rates (see Figs. 9, 11, and 12).

First consider the influence of the dimensionless hotwire conductivity $\sigma_{w}$. Fig. 9 shows the functional relationship $\Delta p(\tau)$ as $\sigma_{w}$ is varied from 0.05 to 0.5 while $\sigma_{d}=1.5$ and $\mathrm{AR}=1$ are fixed. We see that $\Delta p$ is not significantly affected by $\sigma_{w}$, changing only by about $6 \%$ for $\tau$ up to 24 . This agrees with the observation that relations between $\Delta p$ and $\tau$ are virtually



Fig. 9. Dimensionless shear-induced hotwire power $\Delta \mathrm{p}$ as a function of the dimensionless shear stress $\tau$ for different values of the dimensionless hotwire conductivity $\sigma_{w}\left(\sigma_{d}=1.5, \mathrm{AR}=1\right)$.

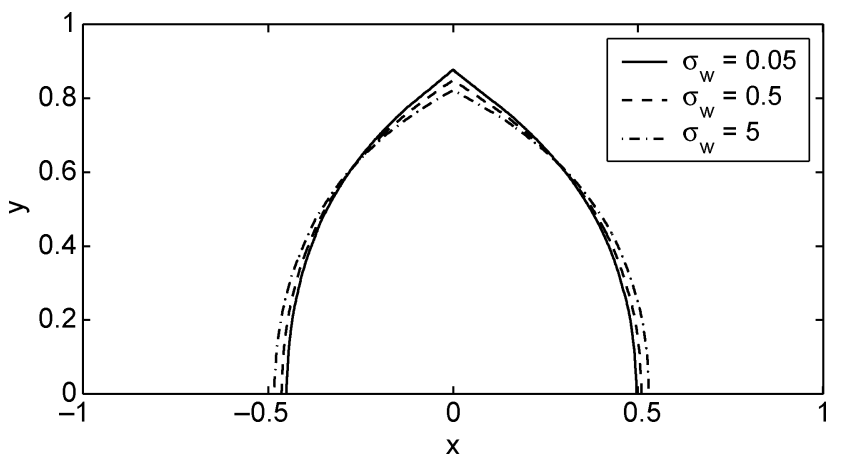

Fig. 10. Boundary of the effective heated region on the diaphragm at $\tau=$ 20 for different values of the dimensionless hotwire conductivity $\sigma_{w}\left(\sigma_{d}=\right.$ $1.5, \mathrm{AR}=1$ ). The bounded region consists of points at which $\theta_{d}>0.5$.

the same in the experimental data (Fig. 3) even though $\sigma_{w}$ is larger for Sensors 2 and 3 than for Sensor 1. To explain the weak dependence of $\Delta p$ on $\sigma_{w}$, we consider the effective heated region, the diaphragm region where the temperature is significantly higher than room temperature (using $\theta_{d}>0.5$ for concreteness). As depicted in Fig. 10 for $\tau=20$ and different $\sigma_{w}$ values, the boundary of this region does not vary significantly with $\sigma_{w}$, as the sensor is in constant-temperature operation. Thus, heat loss from the diaphragm to the fluid, and hence $\Delta p$, is not significantly affected by $\sigma_{w}$.

To study the effect of the dimensionless diaphragm conductivity $\sigma_{d}$, the functional relationship $\Delta p(\tau)$ is plotted in Fig. 11 with $\sigma_{d}$ varying from 0.05 to 5 while $\sigma_{w}=0.5$ and $\mathrm{AR}=1$ are fixed. At a given $\tau, \Delta p$ increases with $\sigma_{d}$, as higher diaphragm conductivities allow for a larger effective heated region and hence increased forced convection rates. However, the heat transfer rate quickly becomes saturated when $\sigma_{d}$ is sufficiently large (approximately $\sigma_{d}>0.5$ ). For example, $\Delta p$ only changes by less than $20 \%$ as $\sigma_{d}$ varies from 0.5 to 5 , and by less than $7.8 \%$ as $\sigma_{d}$ varies from 1 to 3.5. This is again consistent with the experimental data, which gives nearly identical relations between $\Delta p$ and $\tau$ (see Fig. 3) for $\sigma_{d}$ ranging from 1.09 to 3.27. This is because the effective heated region on the diaphragm, 


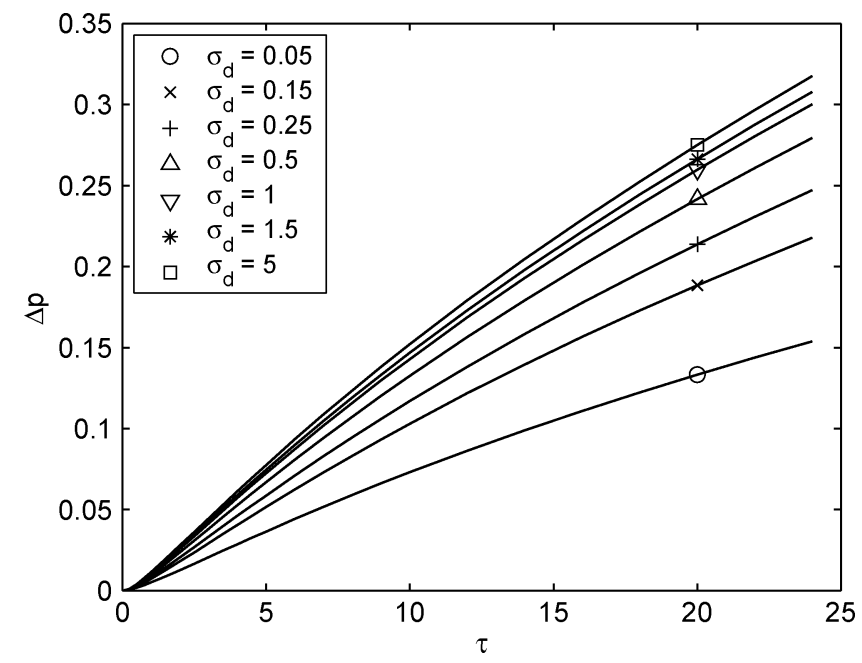

Fig. 11. Dimensionless shear-induced hotwire power $\Delta \mathrm{p}$ as a function of the dimensionless shear stress $\tau$ for different values of the dimensionless diaphragm conductivity $\sigma_{d}\left(\sigma_{w}=0.5, \mathrm{AR}=1\right)$.

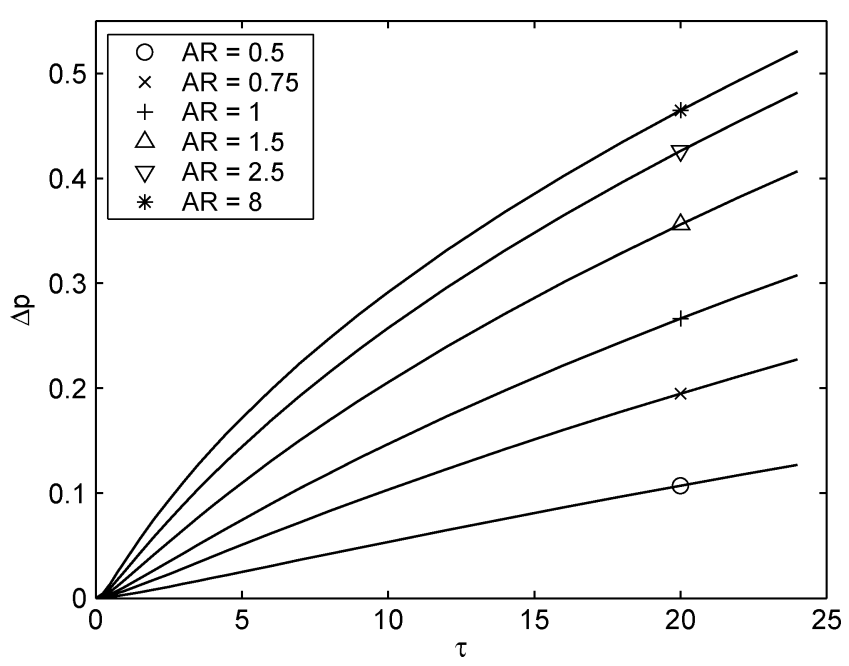

Fig. 12. Dimensionless shear-induced hotwire power $\Delta \mathrm{p}$ as a function of the dimensionless shear stress $\tau$ for different values of the aspect ratio $\mathrm{AR}\left(\sigma_{w}=\right.$ $\left.0.5, \sigma_{d}=1.5\right)$.

in a manner similar to Fig. 10, is not sensitive to sufficiently large $\sigma_{d}$. As $\sigma_{d}$ increases, the temperature distribution on the diaphragm ultimately approaches an asymptote. Thus, $\Delta p$, which is primarily due to heat transfer from the diaphragm surface to the fluid, no longer changes with $\sigma_{d}$ significantly.

Finally, consider the effect of the diaphragm aspect ratio AR. Fig. 12 shows $\Delta p$ as a function of $\tau$ with AR varying from 0.5 to 8 , and $\sigma_{w}=0.5$ and $\sigma_{d}=1.5$. It can be seen that the dependence of $\Delta p$ on $\tau$ is relatively linear at small $\mathrm{AR}$, and becomes increasingly nonlinear as AR increases. For a fixed $\tau, \Delta p$ also increases with AR and eventually approaches an asymptotic value. To understand this trend, we note that the diaphragm's effective heated region initially increases with AR in both streamwise and spanwise dimensions, leading to more efficient convection and increased $\Delta p$. As AR further increases, the growth of the heated region's streamwise dimension becomes saturated, while the heated region's spanwise dimension continues to increase almost proportionally to AR, or the span $B$ with $L$ fixed (see Fig. 2). Thus, similar to $p_{0}$ (see Fig. 6 and 8), (3) implies that $\Delta p$ becomes virtually independent of AR in such a 2-D sensor configuration.

\section{CLOSED-FORM CORRELATIONS}

We seek closed-form correlations to represent the functional relationship $p(\tau)$, which can be further decomposed using (8). For the still-fluid power $p_{0},(5)-(7)$ are numerically solved using parameter combinations $\left(\sigma_{w}\right.$, $\sigma_{d}$, AR) with $\sigma_{w} \in\{0.01,0.05,0.1,0.2,0.5,1,2.5,5\}$, $\sigma_{d} \in\{0.05,0.1,0.15,0.25,0.37,0.5,0.75,1,1.5,2.5,3.25,5\}$, and $\mathrm{AR} \in\{0.5,0.75,1,1.5,2,2.5,4,8\}$. The numerical results are then fitted to the following equation:

$$
p_{0}=a_{0}+a_{1} \sigma_{w}+a_{2} \sigma_{d}+a_{3} \sigma_{w} \sigma_{d} .
$$

This functional form and the specific parameter values above are chosen based on the observation that $p_{0}$ is virtually linear in $\sigma_{w}$ and in sufficiently large $\sigma_{d}$. The coefficients in (10) depend on AR and are found to be

$$
\begin{aligned}
& a_{0}=0.634+\frac{0.443}{\mathrm{AR}^{0.397}}, \quad a_{1}=0.0267+\frac{2.99}{\mathrm{AR}^{2.01}} \\
& a_{2}=2.07+\frac{2.00}{\mathrm{AR}^{1.45}}, \quad a_{3}=0.0119+\frac{0.00743}{\mathrm{AR}^{1.31}}
\end{aligned}
$$

This correlation agrees with numerical results within $1.5 \%$ for $0.05 \leq \sigma_{w} \leq 5,0.2 \leq \sigma_{d} \leq 5$ and $0.5 \leq \mathrm{AR} \leq 8$. The correlation is also valid for a larger range of $\sigma_{d}$ (e.g., within 5\% of numerical results for $0.15 \leq \sigma_{d} \leq 5$ over the same ranges of $\sigma_{d}$ and AR above).

Due to the insensitivity of the dimensionless net hotwire power $\Delta p$ to the dimensionless hotwire conductivity $\sigma_{w}$ (Section VII), we use the typical value $\sigma_{w}=0.5$, and solve (5)-(7) numerically with $\sigma_{d}$ and AR drawn from the same value sets as above. The following analytical expression is then fitted to the numerical results:

$$
\Delta p=\frac{b_{1} \tau^{m}}{1+b_{2} \tau^{n}} .
$$

The choice of this functional form is based on the observation that the slope $\partial(\Delta p) / \partial \tau$ decreases with $\tau$. Given that the dependence of $\Delta p$ on both $\sigma_{d}$ and AR becomes saturated at sufficiently large parameter values, the coefficients in (11) are found to be

$$
\begin{aligned}
& m=1.52, \quad n=0.978-0.441 e^{-1.95 \mathrm{AR}} \\
& b_{1}=\frac{\left[\alpha_{1}+\alpha_{2} \sigma_{d}\right]}{\left[1+\alpha_{3} \sigma_{d}\right]}, \quad b_{2}=\beta_{1}\left[1+\beta_{2} \sigma_{d}\right] \sigma_{d}^{\gamma}
\end{aligned}
$$


and

$$
\begin{aligned}
\alpha_{1} & =0.00621 \mathrm{AR}^{1.70} \frac{\left(1-\frac{0.196}{\mathrm{AR}}\right)}{\left(1+0.265 \mathrm{AR}^{1.67}\right)} \\
\alpha_{2} & =\frac{0.0973 \mathrm{AR}^{1.76}}{\left(1+0.185 \mathrm{AR}^{1.89}\right)} \\
\alpha_{3} & =3.96+1.17 \mathrm{AR}-0.104 \mathrm{AR}^{2}+\frac{0.925}{\mathrm{AR}} \\
\beta_{1} & =0.120+0.100 \mathrm{AR}-0.00692 \mathrm{AR}^{2}+\frac{0.0198}{\mathrm{AR}} \\
\beta_{2} & =-0.806+\frac{13.2 \mathrm{AR}^{0.526}}{\left(1+15.3 \mathrm{AR}^{0.526}\right)} \\
\gamma & =0.223-\frac{0.553 \mathrm{AR}^{1.24}}{\left(1+1.74 \mathrm{AR}^{1.24}\right)}
\end{aligned}
$$

With these coefficients, (11) represents the numerical results with an error smaller than $7.8 \%$ for $1 \leq \tau \leq 24,0.5 \leq \mathrm{AR} \leq 8$ and $0.05 \leq \sigma_{d} \leq 5$.

The above-mentioned ranges of $\tau, \sigma_{w}, \sigma_{d}$ and AR, over which (10) and (11) are obtained, encompass the experimental data discussed in Section IV, and are representative of MEMS shearstress sensors that feature the Fig. 1 configuration and are used in air flow measurements (e.g., [10], [19], [20]). As such, these closed-form and parametrized correlations, which are highly efficient while retaining the accuracy of a 3-D model, are useful in practical iterative design processes to quantitatively evaluate candidate sensor designs.

\section{CONCLUSION}

We have developed a parametrized, 3-D model for MEMS thermal shear-stress sensors that feature a hotwire deposited on a vacuum-isolated diaphragm. The model considers coupled heat transfer in the sensor and yields a functional relationship between the dimensionless hotwire power $p$ and dimensional shear-stress $\tau$. This functional relationship is parametrized by three dimensionless parameters: the dimensionless hotwire and diaphragm thermal conductivities $\sigma_{w}$ and $\sigma_{d}$, and the diaphragm aspect ratio AR. Representing this relationship with closed-form correlations, we have obtained a highly efficient model that retains the accuracy of three-dimensional numerical simulations. This model was validated by wind-tunnel testing results from MEMS shear-stress sensors. It was then used to quantitatively elucidate that MEMS thermal shear-stress sensors do not admit a thin thermal boundary layer for experimentally relevant shearstress levels in air flow, and hence cannot be adequately described by classical theory. The model was also used to systematically study the influence of the dimensionless parameters $\sigma_{w}$, $\sigma_{d}$ and AR on MEMS sensor behavior. It was found that the still-fluid hotwire power $p_{0}$ increases almost linearly with $\sigma_{w}$ and sufficiently large $\sigma_{d}$, and decreases with AR until reaching an asymptotic value. On the other hand, the shear-induced net hotwire power $\Delta p$ is insensitive to $\sigma_{w}$, while increasing with both $\sigma_{d}$ and AR until reaching saturation. In summary, the efficient and accurate model is useful in practical design processes to evaluate the impact of geometry and material choices on sensor performance.
Further work will address physical effects that are not currently included in the model for improved accuracy, especially in the net hotwire power. For example, it would be interesting to investigate sensor models in which the hotwire does not span the entire width of the supporting diaphragm, and to include heat transfer that occurs directly from the hotwire to the fluid. In addition, while it is estimated that the effects of natural convection and radiation are not significant in the current investigation, a more quantitative assessment of such effects is desirable. In particular, consideration of natural convection effects will allow the model to be extended to represent MEMS shear-stress sensors operating in water. These issues should be addressed in a way such that the model complexity is still limited to a tractable level to benefit practical design.

\section{REFERENCES}

[1] B. J. Bellhouse and D. L. Schultz, "Determination of mean and dynamic skin friction, separation and transition in low-speed flow with a thin-film heated element," J. Fluid Mech., vol. 24, pp. 379-400, 1966.

[2] J. H. Haritonidis, "The measurements of wall shear stress," in Advanced Fluid Mechanics Measurements. New York: Springer-Verlag, 1989, pp. 229-261.

[3] S. Goldstein, Fluid Mechanics Measurements, 2nd ed. New York: Hemisphere, 1996.

[4] M. Kimura, S. Tung, J. Lew, C. M. Ho, F. Jiang, and Y. C. Tai, "Measurements of wall shear stress of a turbulent boundary layer using a microshear-stress imaging chip," Fluid Dynam. Res., vol. 24, pp. 329-342, 1999.

[5] P. R. Bandyopadhyay, "Development of a microfabricated surface for turbulence diagnostics and control," in Applied Microfabrication to Fluid Mechanics Chicago, IL, 1994, pp. 67-74.

[6] F. Jiang, "Silicon-Micromachined Flow Sensors," Ph.D. dissertation, California Inst. Technol., Pasadena, CA, 1998.

[7] M. A. Schmidt, R. T. Howe, S. D. Senturia, and J. H. Haritonidis, "Design and calibration of a microfabricated floating-element shear-stress sensor," IEEE Trans. Electron. Devices, vol. 35, pp. 750-757, 1988.

[8] T. Pan, D. Hyman, M. Mehregany, E. Reshotko, and S. Garverick, "Microfabricated shear stress sensors, part 1: design and fabrication," AIAA J., vol. 37, pp. 66-72, 1999.

[9] C. Liu, Y.-C. Tai, J. B. Huang, and C.-M. Ho, "Surface micromachined thermal shear stress sensor," in Proc. ASME Int. Mech. Eng. Congress and Expo., Chicago, IL, 1994, pp. 9-15.

[10] C. Liu, J. B. Huang, A. Zhu, F. Jiang, S. Tung, Y.-C. Tai, and C.-M. Ho, "A micromachined flow shear stress sensor based on thermal transfer principles,” J. Microelectromech. Syst., vol. 8, pp. 90-99, 1999.

[11] F. Jiang, Y.-C. Tai, J. B. Huang, and C.-M. Ho, "Polysilicon structures for shear stress sensors," in Proc. IEEE Region 10 Int. Conf. Microelectronics and VLSI, Hong Kong, 1995, pp. 16-19.

[12] E. Kalvesten, "Pressure and Wall Shear Stress Sensors for Turbulence Measurements," Ph.D. dissertation, Royal Inst. Technol., Stockholm, Sweden, 1996.

[13] L. Lofdahl and M. Gad-el-Hak, "MEMS-based pressure and shear stress sensors for turbulent flows," Meas. Sci. Tech., vol. 10, pp. 665-686, 1999.

[14] A. Cain, V. Chandrasekaran, T. Nishida, and M. Sheplak, "Development of a wafer-bonded, silicon-nitride membrane thermal shear-stress sensor with platinum sensing element," in Tech. Digest, Solid-State Sensor and Actuator Workshop, Hilton Head Island, SC, 2000, pp. 300-303.

[15] X. Q. Wang, Z. Han, F. Jiang, T. Tsao, Q. Lin, Y. C. Tai, V. Koosh, R. Goodman, J. Lew, and C. M. Ho, "A fully integrated shear stress sensor," in Tech. Digest, Int. Conf. Solid-State Sensors and Actuators, Sendai, Japan, 1999, pp. 1074-1077.

[16] J. B. Huang, S. Tung, C.-M. Ho, C. Liu, and Y.-C. Tai, "Improved micro thermal shear-stress sensor," IEEE Trans. Instrum. Meas., vol. 45, 1996.

[17] M. Sheplak, V. Chandrasekaran, A. Cain, T. Nishida, and L. N. Cattafesta, "Characterization of a silicon-micromachined thermal shear-stress sensor," AIAA J., pp. 1099-1104, 2002.

[18] J. B. Huang, F. Jiang, Y.-C. Tai, and C.-M. Ho, "A micro-electro-mechanical-system-based thermal shear-stress sensor with self-frequency compensation," Meas. Sci. Tech., vol. 10, pp. 687-696, 1999. 
[19] F. Jiang, Y.-C. Tai, B. Gupta, R. Goodman, S. Tung, J. B. Huang, and C.-M. Ho, "A micromachined shear stress sensor imager," in Proc. Int. Workshop Micro Electro Mechanical Systems, San Diego, CA, 1996, pp. $110-115$.

[20] F. Jiang, Y.-C. Tai, K. Walsh, T. Tsao, G. B. Lee, and C.-M. Ho, “A flexible MEMS technology and its first application to shear stress sensor skin," in Proc. Int. Workshop Micro Electro Mechanical Systems, Nagoya, Japan, 1997, pp. 465-470.

[21] Y. Xu, Y.-C. Tai, A. Huang, and C.-M. Ho, "IC-integrated flexible shearstress sensor skin," J. Microelectromech. Syst., vol. 12, pp. 740-747, 2003.

[22] T. Tsao, F. Jiang, R. A. Miller, Y.-C. Tai, B. Gupta, R. Goodman, S. Tung, and C.-M. Ho, "An integrated MEMS system for turbulent boundary layer control," in Tech. Digest, Int. Conf. Solid State Sensors and Actuators, Chicago, IL, 1997, pp. 315-318.

[23] Y. Xu, F. Jiang, Q. Lin, J. Clendenen, S. Tung, and Y.-C. Tai, "Underwater shear-stress sensor," in Proc. IEEE Int. Conf. Micro Electro Mechanical Systems, Las Vegas, NV, 2002, pp. 340-343.

[24] A. Appukuttan, W. Shyy, M. Sheplak, and L. Cattafesta, "Mixed convection induced by MEMS-based thermal shear stress sensors," Numer. Heat Transfer, Part A, vol. 43, pp. 283-305, 2003.

[25] D. Meunier, S. Tardu, D. Tsamados, and J. Boussey, "Realization and simulation of wall shear stress integrated sensors," Microelectron. J., vol. 34, pp. 1129-1136, 2003.

[26] Q. Lin, F. Jiang, X. Wang, Y. Xu, Z. Han, Y.-C. Tai, J. Lew, and C.-M. Ho, "Experiments and simulations of MEMS thermal sensors for wall shear stress measurements in aerodynamic control applications," J. Micromech. Microeng., vol. 14, pp. 1640-1649, 2004.

[27] F. P. Incropera and D. P. DeWitt, Fundamentals of Heat and Mass Transfer, 4th ed. New York: Wiley, 1996.

[28] X. Zhang and C. P. Grigoropoulos, "Thermal-conductivity and diffusivity of freestanding silicon-nitride thin-films," Rev. Scientific Instrum., vol. 66, pp. 1115-1120, 1995.

[29] O. Paul, M. von Arx, and H. Baltes, "Process-dependent thermophysical properties of CMOS IC thin films," in Tech. Digest, Int. Conf. Solid-State Sensors and Actuators, and Eurosensors IX, Stockholm, Sweden, 1995, pp. $178-181$.

[30] A. D. McConnell, S. Uma, and K. E. Goodson, "Thermal conductivity of doped polysilicon layers," J. Microelectromech. Syst., vol. 10, pp. 360-369, 2001.

[31] C. H. Mastrangelo, Y. C. Tai, and R. S. Muller, "Thermophysical properties of low-residual stress, silicon-rich, Lpcvd silicon-nitride films," Sens. Actuators A, Phys., vol. 23, pp. 856-860, 1990.

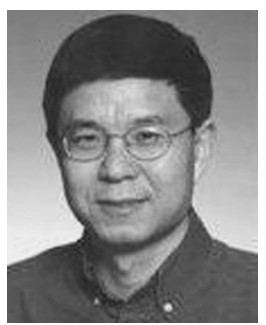

Qiao Lin received the Ph.D. degree in mechanical engineering from the California Institute of Technology (Caltech), Pasadena, in 1998 with dissertation research on kinematics and mechanics of robotic manipulation.

$\mathrm{He}$ conducted postdoctoral research in microelectromechanical systems (MEMS) at the Caltech Micromachining Laboratory from 1998 to 2000, and has since been an Assistant Professor in the Department of Mechanical Engineering at Carnegie Mellon University, Pittsburgh, PA. His research interests are in MEMS, including analysis, design and fabrication of micro/nano-fluidic, thermal, and robotic devices for biological and chemical applications.

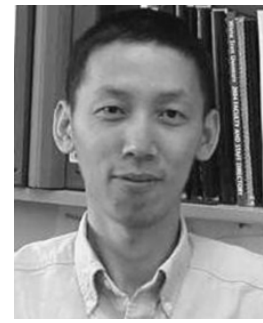

Yong Xu received the B.S. degree in electronics engineering from Tsinghua University, Beijing, China, in 1997 and the M.S. and Ph.D. degrees in electrical engineering from the California Institute of Technology (Caltech), Pasadena, in 1998 and 2002, respectively.

$\mathrm{He}$ is currently an Assistant Professor in the Department of Electrical and Computer Engineering at Wayne State University. His research interests include MEMS smart skins, intelligent textiles, microfluidics, biomedical sensors, novel packaging technology, and nanotechnology.

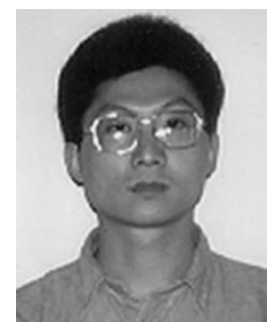

Fukang Jiang received the M.S. and Ph.D. degrees in electrical engineering from the California Institute of Technology in 1992 and 1997, respectively, where his research was on the development of MEMS flow sensors and their advanced packaging technologies such as the flexible-skin-type substrate for MEMS devices.

Since 1997, he has been with Umachines, Inc., developing various optical MEMS products such as optical switches and 3-D scanning mirror arrays.

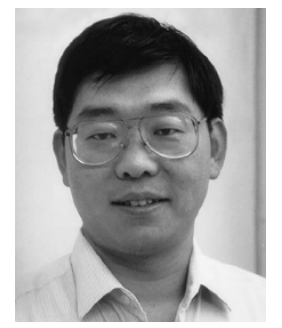

Yu-Chong Tai (M'97) received the B.S. degree from National Taiwan University and the M.S. and Ph.D. degrees in electrical engineering from the University of California at Berkeley

After Berkeley, he joined the faculty of Electrical Engineering at the California Institute of Technology (Caltech), Pasadena, and built the Caltech MEMS Lab. He is currently a full Professor of Electrical Engineering and Bioengineering at Caltech. His research interests include flexible MEMS, MEMS for retinal implants, integrated Parylene nanofluidics, neuroprobes/neurochips and lab-on-a-chip.

Dr. Tai has received several awards such as the IBM fellowship, the Best Thesis Award, the Presidential Young Investigator (PYI) Award and the David and Lucile Packard Fellowship. He Co-Chaired the 2002 IEEE MEMS Conference in Las Vegas. He is currently a Subject Editor of the IEEE/ASME JOURNAL of MiCROELECTROMECHANICAL SYSTEMS.

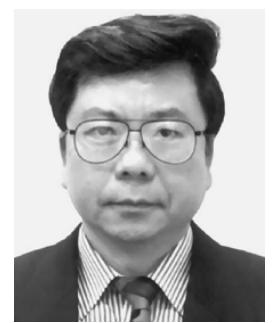

Chih-Ming Ho (M'00) received the Ph.D. degree in mechanics from the John Hopkins University, Baltimore, MD, in 1974.

He currently holds the Ben Rich-Lockheed Martin Professor in the Henry Samueli School of Engineering and serves as the University of California at Los Angeles Associate Vice-Chancellor for Research. He is the Director of the Institute for Cell Mimetic Space Exploration (CMISE). In 1997, he was inducted as a member of the National Academy of Engineering and in 1998, he was elected as an Academician of Academia Sinica.

Dr. Ho is a Fellow of the American Physical Society as well as the American Institute of Aeronautics and Astronautics for his contributions in a wide spectrum of technical areas. 